\title{
Vascular-directed responses of microglia produced by methamphetamine exposure: indirect evidence that microglia are involved in vascular repair?
}

John F. Bowyer ${ }^{1,4^{*}}$, Sumit Sarkar ${ }^{1}$, Karen M. Tranter ${ }^{1}$, Joseph P. Hanig ${ }^{2}$, Diane B. Miller ${ }^{3}$ and James P. O'Callaghan ${ }^{3}$

\begin{abstract}
Background: Brain microglial activations and damage responses are most commonly associated with neurodegeneration or systemic innate immune system activation. Here, we used histological methods to focus on microglial responses that are directed towards brain vasculature, previously undescribed, after a neurotoxic exposure to methamphetamine.

Methods: Male rats were given doses of methamphetamine that produce pronounced hyperthermia, hypertension, and toxicity. Identification of microglia and microglia-like cells (pericytes and possibly perivascular cells) was done using immunoreactivity to allograft inflammatory factor 1 (Aif1 a.k.a Iba1) and alpha M integrin (Itgam a.k.a. Cd11b) while vasculature endothelium was identified using rat endothelial cell antigen 1 (RECA-1). Regions of neuronal, axonal, and nerve terminal degeneration were determined using Fluoro-Jade $C$.

Results: Dual labeling of vasculature (RECA-1) and microglia (Iba1) showed a strong association of hypertrophied cells surrounding and juxtaposed to vasculature in the septum, medial dorsal hippocampus, piriform cortex, and thalamus. The Iba1 labeling was more pronounced in the cell body while Cd11b more so in the processes of activated microglia. These regions have been previously identified to have vascular leakage after neurotoxic methamphetamine exposure. Dual labeling with Fluoro-Jade C and Iba1 indicated that there was minimal or no evidence of neuronal damage in the septum and hippocampus where many hypertrophied Iba1-labeled cells were found to be associated with vasculature. Although microglial activation around the prominent neurodegeneration was found in the thalamus, there were also many examples of activated microglia associated with vasculature.

Conclusions: The data implicate microglia, and possibly related cell types, in playing a major role in responding to methamphetamine-induced vascular damage, and possibly repair, in the absence of neurodegeneration. Identifying brain regions with hypertrophied/activated microglial-like cells associated with vasculature has the potential for identifying regions of more subtle examples of vascular damage and BBB compromise.
\end{abstract}

Keywords: Microglia, Vascular damage, Methamphetamine, Amphetamine, Neurotoxicity, Hyperthermia, Hypertension

\footnotetext{
* Correspondence: John.Bowyer@fda.hhs.gov

'Division of Neurotoxicology, National Center for Toxicology/FDA, Jefferson,

AR 72079, USA

${ }^{4}$ National Center for Toxicological Research/FDA, 3900 NCTR Road, HFT-132,

Jefferson, AR 72079, USA

Full list of author information is available at the end of the article
} 


\section{Background}

Microglia activation responses in connection with neurotoxic insults have been investigated for now over 30 years [1-8]. Microglial responses to METH and amphetamine (AMPH) damage to dopaminergic axons and nerve terminals in the striatum $[9,10]$ and neurodegeneration in the parietal cortex, thalamus, and hippocampus and other limbic regions [11-13] were first identified over 20 years ago. In most cases, it has now been shown that microglia are not involved in producing or exacerbating the neurodegeneration, but responding to it $[2,3,11-13]$. This has been observed for dopaminergic terminal damage in the striatum due to amphetamines or MPTP [14-16]. Most clearly, it was shown that you can block the microglial response without affecting the degree of neurodegeneration, indicating parallel processes [14]. It has been known for some time that microglia play a role in scavenging degenerating neurons [17]. Although the origin and development of microglia in the brain has been elucidated [18], the role of microglia in disease and the neurotoxic process is continually being reevaluated and debated [8, 14, 19-23].

Significant microglia activation can occur just due to systemic signals circulating in the blood such as bacterial inflammagens, lipopolysaccharide (LPS), or damageassociated molecular proteins [24] (DAMPs) that do not cause neurodegeneration $[14,23]$. This raises the possibility that, in regions of the brain where subtle or limited blood-brain barrier (BBB) breakdown or vascular leakage occurs, the activation of microglia and related cells that are adjacent to brain vasculature will occur. Histological signs of BBB breakdown in the septum and medial hippocampus of mouse and rat occur after a single very high dose of METH that can be the "driving force behind" the significant and prolonged signs of seizure activity $[25,26]$. As well, microglial activation via isolectin B4 identification surrounding the vasculature is prominent, particularly in the hippocampus of the mouse brain. However, there is also a significant amount of neurodegeneration in these regions raising the possibility that the microglial response observed may be solely due to neurodegeneration.

Studies have indicated that other paradigms [21, 22] used to produce METH or AMPH neurotoxicity in rat or mouse can produce multiple focal areas (0.25 to $1 \mathrm{~mm}^{2}$ ) of vascular leakage within the thalamus and limbic regions that appear when body temperatures are $\geq 41.7^{\circ} \mathrm{C}[27,28]$. However, behavioral signs of seizure activity are much less pronounced or absent in the paradigm in which four injections (ranging from 5 to $10 \mathrm{mg} / \mathrm{kg}$ ) are spaced over a 6 -h period [26]. As well, although neurodegeneration occurs in the thalamus, in this paradigm, there is usually only minor axonal damage in the more medial and ventral septum and almost no evidence of neurotoxicity in the hippocampus. Therefore, in the present study, we chose this type of dosing paradigm [29] now commonly used to produce METH or amphetamine toxicity, which consisted of giving four injections of $5,7.5,10$, and $10 \mathrm{mg} / \mathrm{kg}$ of $\mathrm{METH}$ with $2 \mathrm{~h}$ in between each injection to see if there was significant microglial activation in the septum, hippocampus, and thalamus and associated vasculature.

\section{Methods \\ Animals}

This study was carried out in accordance with the declaration of Helsinki and the Guide for the Care and Use of Laboratory Animals as adopted and promulgated by the National Institutes of Health. The use of animal testing in this study was done under protocols E7295 and E7519 (issued to John Bowyer) that were approved by the NCTR institutional animal care and use committee (IACUC) which is fully accredited (Food and Drug Administration-National Center for Toxicological Research Accreditation \#A4310-01) by NIH-OLAW. Sixtyfive-day-old (300-350 g) male Sprague-Dawley rats, ninety-five total, were obtained from the Charles River Laboratories [Crl:CD(SD)]. Upon arrival at NCTR, they received tail tattoos for identification. Prior to testing, rats were housed 2 per cage with food and water available ad libitum. Rats were housed on a daily 12-h light cycle with lights on at 6:00 am and off at 6:00 pm. During housing, the temperature $\left(23{ }^{\circ} \mathrm{C}\right)$ and humidity (53\%) were controlled. The rats were tested between 85 and 90 days (12 weeks) of age.

\section{Administration of METH}

Dosing with METH commenced between 7:30 and 8:00 a.m. and ended between 1:30 and 2:00 p.m. Rats were individually housed during testing, and their wood chip bedding was replaced with absorbent pads to keep the METH animals from ingesting the wood chips. Animals were given either four injections (each spaced by 2-h intervals) of $1 \mathrm{ml} / \mathrm{kg}$ saline s.c. or four injections of $\mathrm{METH}$ $(5,7.5,10$, and $10 \mathrm{mg} / \mathrm{kg}$ s.c. spaced at 2-h intervals $)$ at an environmental temperature of $22.5{ }^{\circ} \mathrm{C}$. The D-METH (D-methamphetamine $\mathrm{HCl}$; Sigma-Aldrich, St. Louis, MO) was dissolved in normal saline. The behavior and body temperature of all animals in all groups were monitored at least every hour during testing until at least $3 \mathrm{~h}$ after the last/fourth injection (time of sacrifice). A flexible probe was inserted approximately $7 \mathrm{~cm}$ into the colon to measure body temperature. The animals given saline at $22.5{ }^{\circ} \mathrm{C}$ remained normothermic. The lethal effects of hyperthermia in the METH group, when body temperatures exceeded $41.6{ }^{\circ} \mathrm{C}$, were prevented by placing the animals unrestrained on crushed ice for 15 to 
30 min in a clean, wood chip-free cage to allow their temperatures to drop below $40.0{ }^{\circ} \mathrm{C}$.

\section{Animal perfusions and preparation for histological analysis} The perfusion process necessary for histological processing was initiated by giving the rats a lethal dose of approximately 150 to $300 \mathrm{mg} / \mathrm{kg}$ i.p of pentobarbital and 20 to $40 \mathrm{mg} / \mathrm{kg}$ sodium phenytoin derived from a mixture of Euthasol ${ }^{\circ}$ from Vibrac AH, Inc., containing in $390 \mathrm{mg} / \mathrm{ml}$ pentobarbital and $50 \mathrm{mg} / \mathrm{ml}$ sodium phenytoin diluted 1:4 in sterile normal saline. When the rat's respiration had waned to that barely detectable, it was perfused with $50 \mathrm{ml}$ saline followed by $200 \mathrm{ml}$ of $4 \%$ formaldehyde in $0.1 \mathrm{M}$ sodium phosphate buffer $(\mathrm{pH}$ 7.4). Details of the perfusion process have been previously reported [11]. Brains were postfixed for $36 \mathrm{~h}$ in $4 \%$ formaldehyde in $0.1 \mathrm{M}$ sodium phosphate buffer and then transferred to a $20 \%$ sucrose and $0.1 \mathrm{M}$ sodium phosphate buffer for at least 3 days. Sectioning of the brain to produce 25 to $30 \mu \mathrm{m}$ sections was performed either using unfrozen fixed brain as previously described [11] or 20- to $25-\mu \mathrm{m}$-thick sections using a cryostat. For cryostat sectioning, the brain was removed from the sucrose and rapidly frozen in crushed/powdered dry ice. It was then embedded in OCT compound (Electron Microscope Science, Hatfield, PA, USA) and mounted on a Leica Cryostat for cutting coronal sections 20 - to $25-\mu \mathrm{m}$ thick from +1.2 to -6.0 relative to the Bregma [30]. Approximately half of the sections were stored at $4{ }^{\circ} \mathrm{C}$ in $0.1 \mathrm{M}$ phosphate buffer $\mathrm{pH} 7.4$ containing $4 \%$ formalin until histological processing. The remaining sections were in stored $0.1 \mathrm{M}$ phosphate buffer $\mathrm{pH} 7.4$ containing $0.08 \%$ sodium azide at $4{ }^{\circ} \mathrm{C}$ to avoid loss of immunoreactivity or antigens present in the tissue.

\section{Immunohistological and Fluoro-Jade C labeling Fluoro-Jade C labeling}

Methods of Schmued et al. [31] with minor modifications were used to detect degenerating neurons, dendrites, axons, and terminals in the forebrain using Fluoro-Jade C (FJc). The modifications made results in a slightly higher background, which increases the possibility of detecting a false positive signal, but this was deemed necessary to enable dual labeling of FJc with DAB immune-labeling of microglia or vasculature. Sections, either unprocessed or previously immune-labeled, were mounted on gelatin (Sigma, 300Bloom)-coated slides and dried at $50{ }^{\circ} \mathrm{C}$. They were then transferred sequentially through solutions of $100 \%$ ethanol $(8 \mathrm{~min})$, $95 \%$ ethanol $(2 \mathrm{~min}), 70 \%$ ethanol $(2 \mathrm{~min})$ and two times double-deionized water $(2 \mathrm{~min})$. They were then immersed in double distilled water containing $0.06 \%$ potassium permanganate (4 to $6 \mathrm{~min}$ for immune- labeled section or 8 to 10 min for unprocessed sections, followed by two times double-deionized water $(2 \mathrm{~min})$. Labeling was performed in a $0.0001 \%$ FJc (Histo-Chem, Jefferson, AR, USA) in $0.1 \%$ acetic acid and distilled water solution followed by rinsing in double distilled water three times (1 min per wash) to remove excess label. Slides were then rapidly air-dried, xylene-cleared and cover-slipped with DPX (Fluka/Sigma, St. Louis, Mo, USA) mounting media. In instances where sections were double labeled with FJc and Iba-1 or Cd11b, the Iba- 1 and Cd11b labeling with DAB was performed first after which the sections were mounted on the gelatin slides and processed with FJc. The only modification of the procedure for double labeling was that the sections were exposed to the permanganate solution for only $4 \mathrm{~min}$.

\section{Immunohistochemical labeling}

For immune-reactive labeling of brain sections, either single or double, the following procedures were used. In all steps the sections, whether mounted on slides or free-floating in incubation wells, were gently agitated on an orbital shaker. Sections were first washed $15 \mathrm{~min}$ in $0.1 \mathrm{M}$ phosphate buffer $\mathrm{pH}$ 7.4. In cases where diaminobenzedine (DAB) was used as the chromophore, the sections were then incubated in phosphate buffer containing $0.3 \% \mathrm{H}_{2} \mathrm{O}_{2}$ for $30 \mathrm{~min}$ to destroy the endogenous peroxidases. From this point on, except with the last step of $\mathrm{DAB}$ processing the incubation and washing solutions used consisted of $0.1 \mathrm{M}$ phosphate buffer pH7.4 containing $0.4 \%$ Triton X-100 in double distilled water. The sections were subsequently washed for $5 \mathrm{~min}$. After a 30-min preincubation in $4 \%$ normal serum (from the animal that the secondary antibody was raised in), the sections were incubated in normal serum and the primary antibody for 1 to $2 \mathrm{~h}$ followed 18 to $24 \mathrm{~h}$ at $5{ }^{\circ} \mathrm{C}$. The sections were then washed three times for $10 \mathrm{~min}$ and incubated in secondary antibody for 1 to $2 \mathrm{~h}$. In cases where only single labeling for Iba1 was performed, the signal was amplified using the avidin and biotinylated horseradish peroxidase macromolecular complex (ABC, Vector Laboratories, Burlingame, $\mathrm{CA}$ ) and visualized with $0.4 \mathrm{mg} / \mathrm{ml}$ of 3,3'-diaminobenzidine (DAB) in $50 \mathrm{mM}$ Tris-buffer. The following primary antibodies used were rabbit anti-Iba1, Wako Inc., Japan; rabbit anti-RECA-1 from Abcam, Cambridge, USA; and mouse anti-rat Cd11b, Abd Serotek, USA. The secondary antibodies used were biotinylated goat anti-rabbit, Vector Laboratories, Burlingame, CA, and biotinylated donkey anti-rabbit, Jackson Immunoresearch, Philadelphia, PA).

\section{Combined RECA-1 DAB and Iba1 TRITC fluorescent immunohistochemistry}

DAB immunolabeling of RECA-1 was performed first, as described above, using a biotinylated goat anti-rabbit 
secondary antibody. Following DAB reaction, the sections were washed $3 \times(15 \mathrm{~min} /$ wash $)$ in buffer. Sections were then pre-incubated with $10 \%$ normal horse serum (GIBCO, USA) for $30 \mathrm{~min}$ and then incubated with the primary rabbit anti-Ibal antibody for $2 \mathrm{~h}$ at room temperature followed by $18 \mathrm{~h}$ at $5{ }^{\circ} \mathrm{C}$. Sections were then washed $3 \times$ followed by incubation in a secondary biotinylated donkey anti-rabbit IgG (1:200; Jackson Immunoresearch, Philadelphia, PA) for 1 to $2 \mathrm{~h}$. Subsequently, they were washed three times and incubated in a Streptavidin-TRITC (1:250, Jackson Immunoresearch, Philadelphia, PA) for $2 \mathrm{~h}$ at room temperature. Sections were then washed two times (5 min per wash) and mounted on slides dried. Finally, the slides were cleared in xylene and cover-slipped with DPX mounting medium. Methods very similar to above were used for DAB immunolabeling of Iba1 and TRITC immunolabeling of GFAP to visualize microglia and astrocyte morphology after METH in Fig. 9. GFAP immunolabeling was performed using a polyclonal rabbit anti-GFAP primary antibody (Dako/Agilent Technologies, USA).

\section{Combined RECA-1 and Cd11b immunohistochemistry}

Co-localization of integrin alpha $\mathrm{M}$ (complement component 3 receptor 3 subunit) a.k.a Cd11b was also used to identify macrophages in association with vascular endothelia. DAB immunolabeling of CD11b was performed first described in the previous section using a biotinylated donkey anti-mouse secondary antibody. Following DAB reaction, the sections were washed for $5 \mathrm{~min}$ in Tris-buffer followed by washing in Tris-buffer saline (TBS). Sections were then pre-incubated with $10 \%$ normal horse serum (GIBCO, USA) made in TBS for $30 \mathrm{~min}$ and then incubated with the primary mouse RECA-1 antibody for $24-48 \mathrm{~h}$ at $4{ }^{\circ} \mathrm{C}$. After $48 \mathrm{~h}$, tissue sections were rinsed in TBS containing Triton-X for 3$5 \mathrm{~min}$ for three times followed by incubation in biotinylated secondary antibody (1:200 dilution) for $2-3 \mathrm{~h}$ at RT using TBS as antibody diluent. Then sections were washed in TBS containing Triton-X for 3-5 min, and incubated in Streptavidin-Alkaline Phosphatase (1:200; Promega, USA) for $2 \mathrm{~h}$ are RT. Then tissue sections were rinsed in TBS containing Triton-X for 3-5 min for

Table 1 Summary of hyperthermia, reactive microglia, and neurodegeneration after METH exposure

\begin{tabular}{|c|c|c|c|c|c|c|c|c|c|c|c|}
\hline \multirow[b]{3}{*}{ Rat ID } & \multirow[b]{3}{*}{$\begin{array}{l}\text { Time point } \\
\text { (days) }\end{array}$} & \multirow[b]{3}{*}{ Peak BT $\left({ }^{\circ} \mathrm{C}\right)$} & \multirow[b]{3}{*}{ Times $\mathrm{BT} \geq 41.7^{\circ} \mathrm{C}$} & \multicolumn{8}{|c|}{ Brain regions } \\
\hline & & & & \multicolumn{2}{|l|}{ Septum } & \multicolumn{2}{|c|}{ Hippocampus } & \multicolumn{2}{|c|}{ Thalamus } & \multicolumn{2}{|c|}{ Parietal cortex } \\
\hline & & & & $\begin{array}{l}\text { a FJC } \\
\text { labeling }\end{array}$ & $\begin{array}{l}{ }^{\mathrm{b}} \text { Activated } \\
\text { microglia }\end{array}$ & $\begin{array}{l}{ }^{\mathrm{a}} \mathrm{FJc}^{+} \\
\text {labeling }\end{array}$ & $\begin{array}{l}{ }^{\mathrm{b}} \text { Activated } \\
\text { microglia }\end{array}$ & $\begin{array}{l}{ }^{c}{ }_{F J C} \\
\text { labeling }\end{array}$ & $\begin{array}{l}{ }^{\mathrm{b}} \text { Activated } \\
\text { microglia }\end{array}$ & $\begin{array}{l}{ }^{c}{ }^{{ }_{F} J C} \\
\text { labeling }\end{array}$ & $\begin{array}{l}{ }^{\mathrm{b}} \text { Activated } \\
\text { microglia }\end{array}$ \\
\hline FG278 & 3ys & 41.6 & - & - & - & - & - & - & - & 1 & - \\
\hline FG279 & 3 & 41.0 & - & - & - & - & - & - & - & 1 & - \\
\hline FG230 & 3 & 42.0 & $2 x$ & - & - & - & - & - & - & 4 & - \\
\hline FG288 & 3 & 41.9 & $3 x$ & - & - & - & - & 20 & ++ & 2 & - \\
\hline FG227 & 3 & 42.2 & $3 x$ & + & +++ & - & +++ & 17 & + & 8 & + \\
\hline$F G 268$ & 3 & 42.6 & $3 x$ & - & - & - & - & 3 & - & 10 & + \\
\hline FG298 & 3 & 42.0 & $3 x$ & - & + & - & + & 5 & + & 10 & + \\
\hline FG289 & 3 & 42.7 & $4 x$ & - & - & - & + & 38 & +++ & 9 & + \\
\hline FG269 & 3 & 42.6 & $4 x$ & - & + & - & + & 27 & ++ & 2 & - \\
\hline$F G 228$ & 3 & 42.3 & $5 x$ & + & +++ & - & ++++ & 132 & ++++ & 7 & + \\
\hline$F G 267$ & 3 & 42.3 & $5 x$ & + & ++++ & - & ++++ & 37 & +++ & 4 & + \\
\hline FG291 & 3 & 42.2 & $5 x$ & + & ++++ & - & ++++ & 91 & ++++ & 12 & + \\
\hline FG311 & 3 & 42.4 & $5 x$ & + & +++ & - & ++ & 53 & +++ & 7 & + \\
\hline FG258 & 1 & 41.9 & $2 x$ & - & - & - & - & 0 & - & 3 & - \\
\hline FG259 & 1 & 42.3 & $3 x$ & - & - & - & - & 6 & - & 6 & - \\
\hline FG260 & 1 & 42.1 & $3 x$ & - & - & - & - & 0 & - & 3 & - \\
\hline FG310 & 1 & 42.2 & $4 x$ & $+?$ & $+?$ & $+?$ & $+?$ & 53 & - & 6 & - \\
\hline
\end{tabular}

Data relating to neurodegeneration or microglia activation for the four control animals is not shown since it was all negative. The correlation between the numbers of times body temperature was $\geq 41.7^{\circ} \mathrm{C}$, and the relative number of activated microglia within a region was determined using the Spearman rank-order correlation method. The correlation coefficients were as follows: septum, $r=0.781, p=0.0008$; hippocampus, $r=0.850, p<0.00001$; thalamus, $r=0.927, p<0.00001$; parietal cortex, $r=0.652, p<0.014$. The correlation between the numbers of times body temperature was $\geq 41.7{ }^{\circ} \mathrm{C}$ and the relative number of FJC within a region was determined using the Pearson product-moment correlation. The correlation coefficients were as follows: thalamus, $r=0.714, p=0.0061 ;$ parietal cortex, $r=0.546, p=0.054$ not significant

${ }^{\mathrm{a} F J c}$ positive fibers ( $>10$ per $25-\mu \mathrm{m}$ section per hemisphere) observed in the medial septum

${ }^{\mathrm{b}}$ Relative number of activated lba1 immunoreactive cells/microglia per $25-\mu \mathrm{m}$ section per hemisphere such that per region per hemisphere: $(-)$ indicates $<5$ cells per region; $(+) 5$ to 20 cells; $(++) 20$ to 100 cells; $(+++)>100$ cells

${ }^{\mathrm{C}} \mathrm{FJC}$ positive cells per $25-\mu \mathrm{m}$ section per hemisphere observed in thalamus or parietal cortex 
three times. Sections were incubated in substrate for alkaline phosphatase (nitro blue tetrazolium; ready to use) for 1-2 $\mathrm{min}$, and color reaction was monitored under microscope. Once the reaction was complete, the tissue sections were rinsed in TBS containing Triton-X for 3$5 \mathrm{~min}$ for three times. Subsequently, sections were mounted in distilled water containing $0.1 \mathrm{M} \mathrm{PB}$, dried in warmer, cleared in Xylene, cover-slipped using special mounting medium (H-5000; Vector laboratories), and dried overnight under the hood.

\section{Combined Cd11b and IBA-1 immunohistochemistry}

Co-localization of integrin alpha M (complement component 3 receptor 3 subunit) a.k.a Cd11b was also used to identify macrophages in association with another microglial marker IBA1. DAB immunolabeling of CD11b was performed first described in the previous section using a biotinylated donkey anti-mouse secondary antibody. Following DAB reaction, the sections were washed for $5 \mathrm{~min}$ in Tris-buffer followed by washing in Tris- buffer saline (TBS). Sections were then pre-incubated with $10 \%$ normal horse serum (GIBCO, USA) made in TBS for $30 \mathrm{~min}$ and then incubated with the primary rabbit IBA-1 antibody for $24-48 \mathrm{~h}$ at $4{ }^{\circ} \mathrm{C}$. After $48 \mathrm{~h}$, tissue sections were rinsed in TBS containing Triton-X for 3-5 $\mathrm{min}$ for three times followed by incubation in biotinylated secondary antibody (1:200 dilution) for $2-3 \mathrm{~h}$ at RT using TBS as antibody diluent. Then sections were washed in TBS containing Triton-X for 3-5 min, and incubated in Streptavidin-Alkaline Phosphatase (1:200; Promega) for $2 \mathrm{~h}$ are RT. Then tissue sections were rinsed in TBS containing Triton-X for 3-5 min for three times. Sections were incubated in substrate for alkaline phosphatase (nitro blue tetrazolium; ready to use) for 1$2 \mathrm{~min}$, and color reaction was monitored under microscope. Once the reaction was complete, the tissue sections were rinsed in TBS containing Triton-X for 3-5 min for three times. Subsequently, sections were mounted in distilled water containing $0.1 \mathrm{M} \mathrm{PB}$, dried in warmer, cleared in Xylene, cover-slipped using special mounting medium

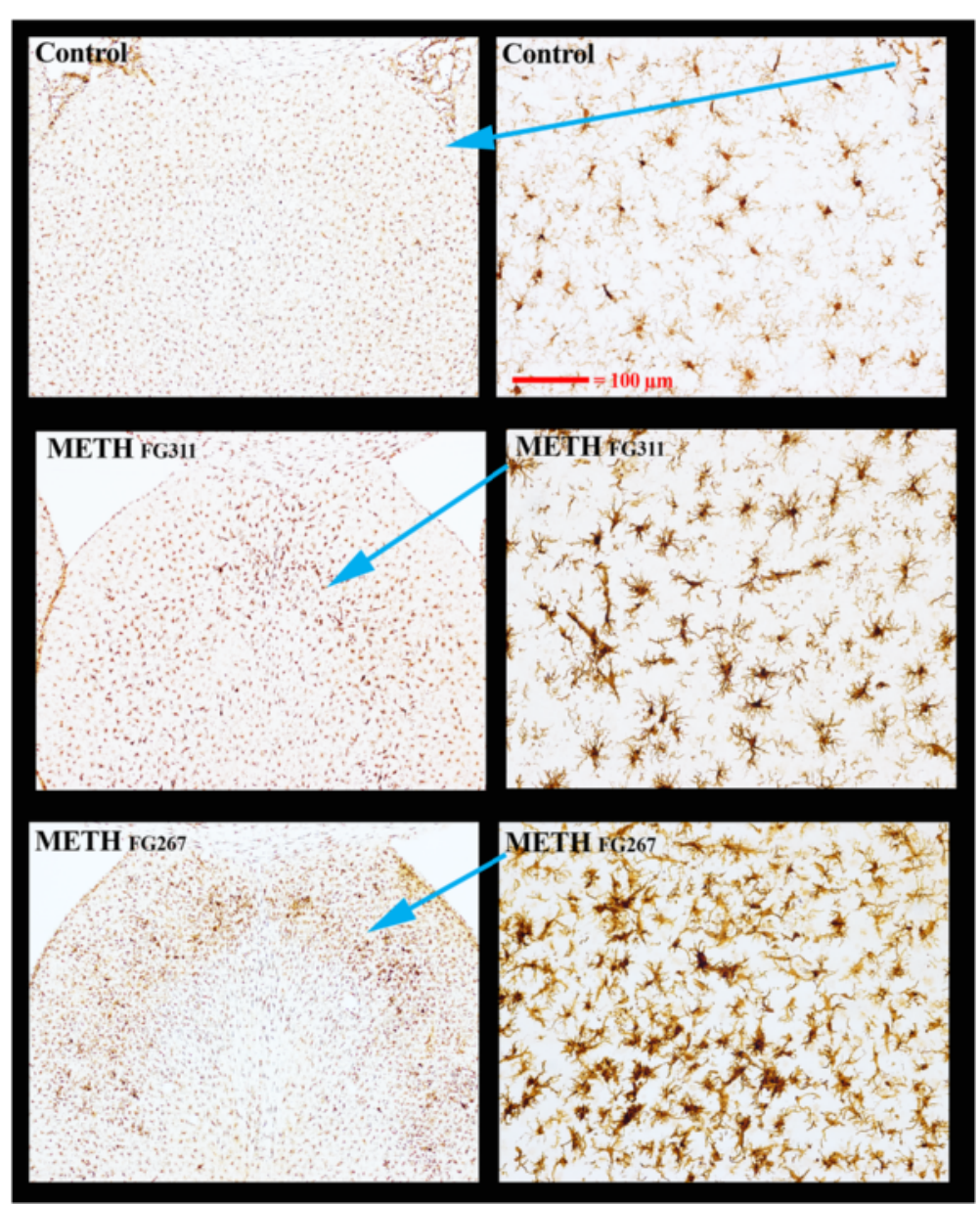

Fig. 1 Microglia activation in the septum at 3 days after METH. Septal microglia were DAB immunolabeled for IBA1 and are shown at low (left-most panels) and high magnification (right-most panels). Magnification for the three right-hand panels was the same (see magnification bar on the top for reference). The blue arrows show where the regions of high magnification reside in the low magnification panels 
(H-5000; Vector laboratories), and dried overnight under the hood.

\section{Visualization}

Histological examination of the slide-mounted tissue was examined using a Nikon epifluorescent microscope 80i (Nikon Instruments Inc., Melville, NY) and an X-Cite 120 LED light source (Excelitas Technologies, Waltham, MA). The following sets of filters were used for visualization fluorescent labeling in conjunction with the histochemical or immunohistological labeling: TRITC excitation of 533$553 \mathrm{~nm}$ and an emission of 573-613 nm and FITC excitation of 464.5-499.5 and an emission of 516-556 nm. Combinations of these filters resulted in negligible cross-talk between the signals from individual fluorochromes, such that there was minimal to no observable bleed-through between any of the three signals. Visualization of DABlabeled sections was done with incandescent illumination.
All photomicrographs of the images were taken using a Nikon DS-Ri1 using the NIS software from Nikon.

\section{Results}

Seventeen of the 19 animals dosed with METH and sacrificed at the 3 days time point had body temperatures $\geq 40.0{ }^{\circ} \mathrm{C}$ for $4 \mathrm{~h}$ or more and at least two episodes of body temperatures $\geq 41.7^{\circ} \mathrm{C}$ requiring hypothermic intervention/cooling (Table 1). The remaining two animals had body temperatures $\geq 40.0^{\circ} \mathrm{C}$ for $4 \mathrm{~h}$ or more but their peak body temperatures did not reach $41.7^{\circ} \mathrm{C}$, and thus they did not requiring cooling. The survival rate was approximately $90 \%$ for the METH-treated animals. As expected with this type of dosing paradigm originally developed to in rodent to produce pronounced and prolonged striatal dopamine depletions and terminal damage/ destructions due to METH (or amphetamine) exposure [29, 32-35], histological evidence of dopamine terminal damage and destruction were pronounced (data not shown).

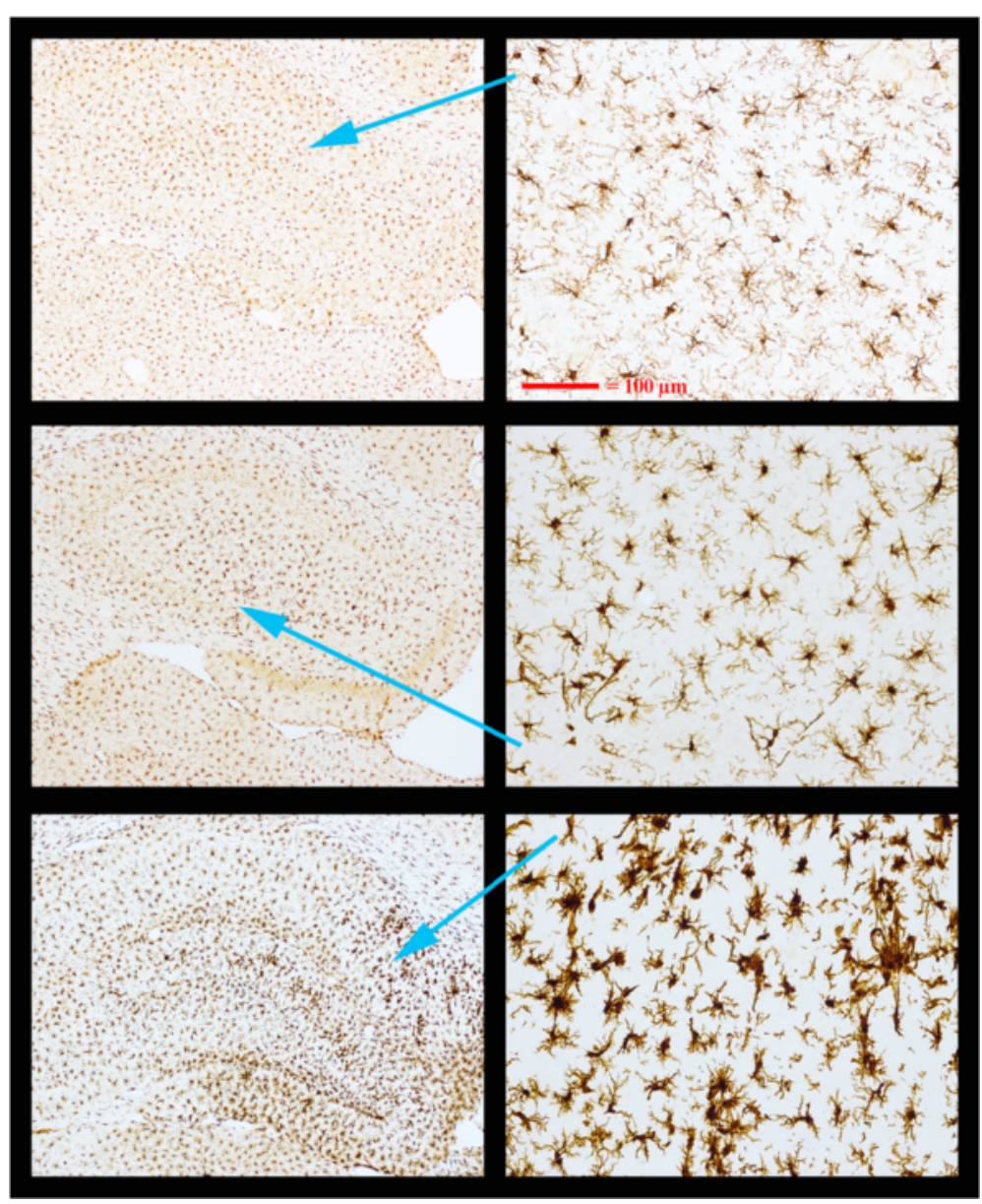

Fig. 2 Microglia activation in the hippocampus at 3 days after METH. The microglia located in the more rostral and dorsal-medial regions of the hippocampus were DAB immunolabeled for IBA1 and are shown at low (left-most panels) and high magnification (right-most panels). Magnification for the three right-hand panels was the same (see magnification bar on the top for reference). The blue arrows show where the region of high magnification resides in the low magnification panels 
Iba1 immunoreactivity, which is an often used biomarker for microglia [36], was used to detect microglia in the septum, medial hippocampus, thalamus, and parietal cortex. The presence of activated microglia and FJcpositive $\left(\mathrm{FJc}^{+}\right)$cells in four brain regions are shown in Table 1. Both activated and resting microglia can be clearly and distinctly labeled by Iba1 immunoreactivity; those that are activated have a greatly enlarged soma, and often, the processes emanating from the soma are enlarged but of lesser length. For animals sacrificed 3 days after METH exposure, the number of Iba1 immunoreactive activated (based on criteria stated above) microglia in the dorsal lateral septum, anterior medial hippocampus, and the intralaminar, ventrolateral (VL), and ventromedial (VM) thalamus nuclei correlate positively with the number of times the body temperature of an animal exceeded $41.7^{\circ} \mathrm{C}$ (see Table 1 for details). As well, the number of $\mathrm{FJc}^{+}$cells in the thalamus and $\mathrm{FJc}^{+}$ fibers in the septum correlated with episodes of body temperatures $\geq 41.7{ }^{\circ} \mathrm{C}$, which was not the case for the number of $\mathrm{FJc}^{+}$cells present in the parietal cortex.

The morphology of the majority of the activated microglia in the parietal cortex was of the hypertrophied spherical soma (with concomitant loss of long fibrous looking processes) type previously identified as being associated with degenerating neurons [2] (data not shown). However, there were some activated microglia in this region with the morphology of the predominant type seen in the septum and hippocampus (see below). The total number of activated microglia in the more dorsal and lateral septum (Fig. 1 and Additional file 1: Figure S1A) and anterior medial hippocampus (Fig. 2 and Additional file 1: Figure S2A) was in much greater numbers. In addition, the morphology of these activated microglia with greatly enlarged somas and significantly reduced processes, and most were "tubular" or "worm-like" not spherical. Thus, they did not have the appearance of activated microglia normally associated with neurodegeneration.

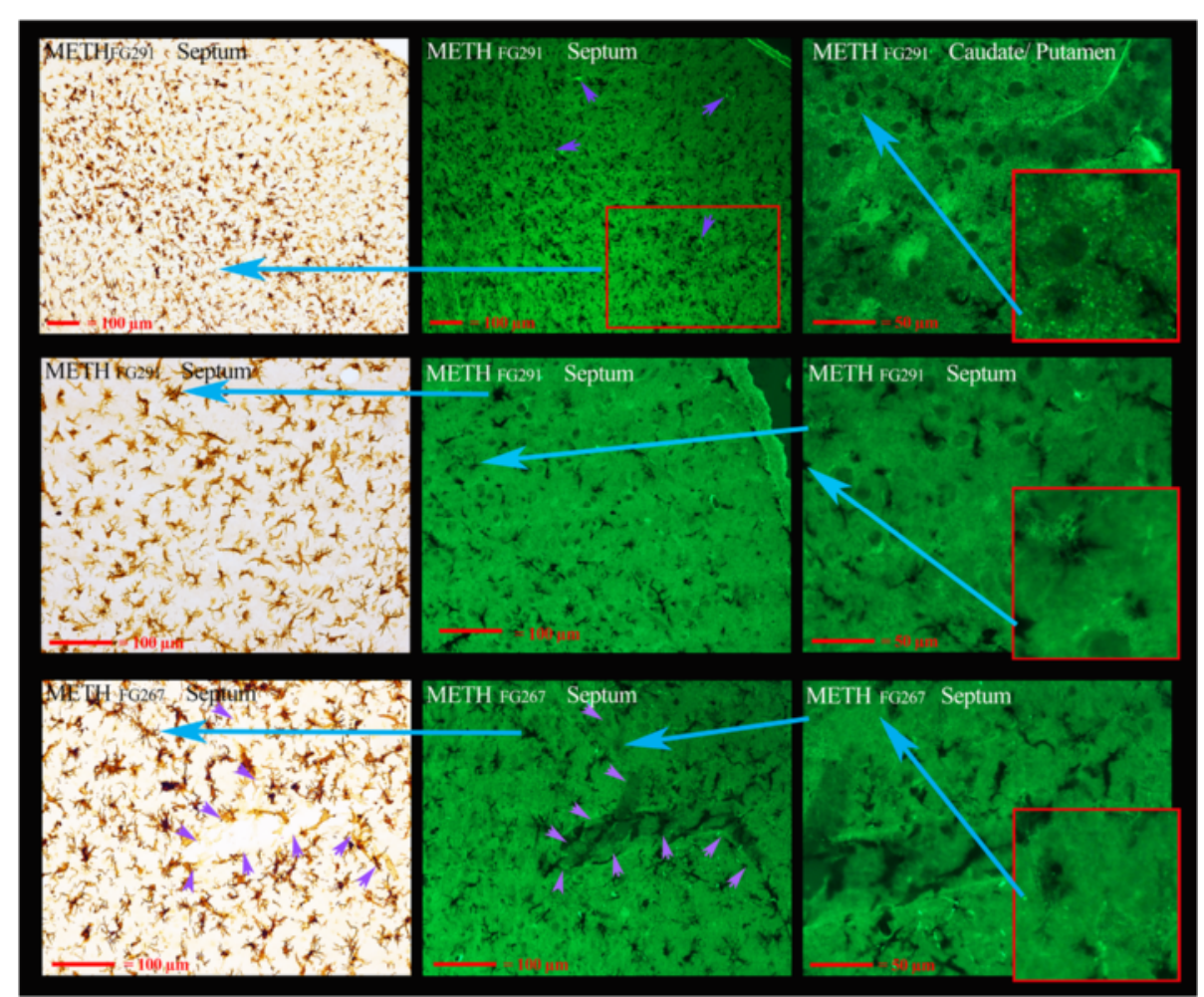

Fig. 3 Pronounced microglia activation in the septum with little or no apparent neurodegeneration. Dual labeling of microglia with IBA1 immunoreactivity and FJc to detect neurodegeneration is shown in the dorsal and more lateral aspects of the septum. The three left-hand panels show the DAB-labeled microglia with incandescent illumination while the three center, and three most right-hand panels are fluorescent images obtained using a TRITC filter (for FJc detection of neurodegeneration). The top left and center panels from a METH-treated animal ( 3 days post dosing) show that there is pronounced microglia activation with minimal evidence of neurodegeneration. The most intensely labeled structures in these dorsal lateral regions, indicated by purple arrows, are vasculature-related. The top right-hand panel shows the FJc-labeled degenerating puncta in the caudate putamen at high magnification of the same animal. The remaining six panels are from two different METH-treated animals that further show there is little or no FJc evidence of neurodegeneration in many of the dorsal septal regions with activated microglia. The purple arrows in two of the bottom panels outline a large vessel that is present. The blue arrows show where the region of high magnification resides in the low magnification panels 
The four animals sacrificed at the 1-day time point showed minimal or no clear classic evidence of Iba1 immunoreactive activated microglia in the septum or hippocampus (Table 1 and Additional file 1: Figure S1B) or the hippocampus or thalamus (Table 1 and Additional file 1: Figure S2B). This, despite the fact that in two of the animals, there was FJc evidence of significant neurodegeneration at the 1-day time point (Table 1), is a time point that significantly underestimates the actual number of neurons compared to the 3-day time point (Bowyer et al. unpublished, [26]). Only one of the four animals showed any early evidence microglia activation in the septum with either Iba1 or Cd11b [36] (biomarker for microglia) or soma enlargement (data not shown). In contrast, in the caudate putamen, all four of the animals showed clear evidence that microglial activation was underway at 1 day post METH (Additional file 1: Figure S2B) which has been previously reported by several laboratories [16, 37-39]. There was virtually no evidence in any brain region, in any of the four animals, examined at either the 1 or 3 days time points, of Iba1 or Cd11b immune-reactive macrophages invading the CNS or intercalating/invading into the brain vasculature.

FJc labeling in the cortex and particularly the thalamus revealed the loss of cell bodies (neurons) and their dendrites and axonal-like process/terminals (Table 1, Additional file 1: Figure S3A). In the ventral and more medial septum, there was evidence of $\mathrm{FJc}^{+}$fibers of passage but very few (ventral medial septum) or no instances of FJc labeling indicating cell body loss (Additional file 1: Figure S3B). Dual labeling for Iba1 immunoreactivity and FJC indicated that most of the activated microglia immunoreactive for Iba1 that were present in the dorsal and central lateral septum were found in regions in which there was no FJc labeling (Fig. 3). In fact, there were areas in the central/mid-septum with significant FJc fiber labeling where the no juxtaposed activated microglia are found.

There was little or no FJc labeling in the hippocampus that could be associated with any type of neurodegeneration in any of the animals (Additional file 1: Figure S4). Figure 4 shows dual labeling for FJc and Iba1 immunoreactivity in the anterior medial hippocampus as well. We modified the original methods of Schmued et al.

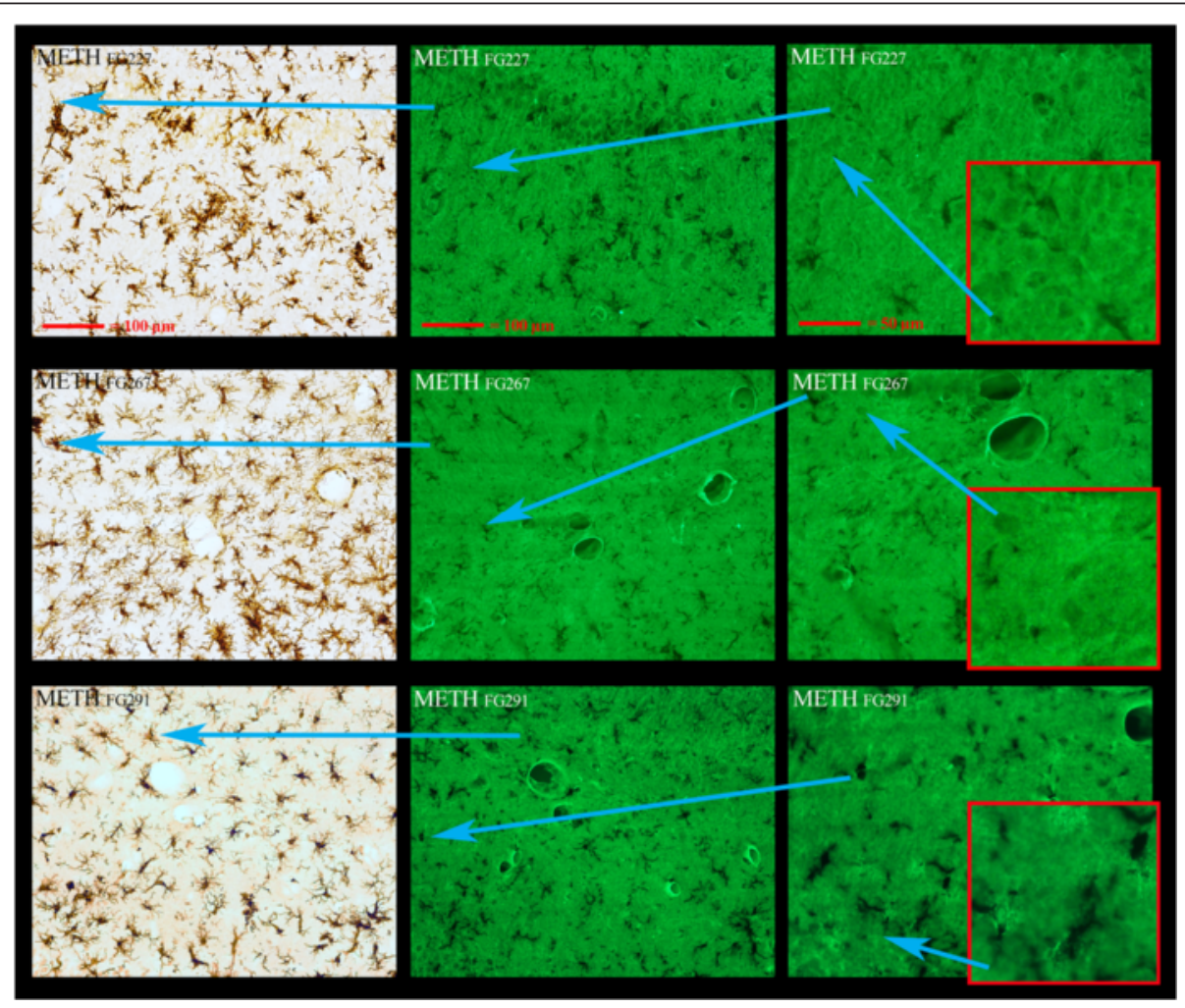

Fig. 4 Pronounced microglia activation occurs with little or no apparent neurodegeneration. Dual labeling of microglia with IBA1 immunoreactivity and FJc to detect neurodegeneration is shown in the rostral and dorsal-medial area of the hippocampus. Pronounced microglial activation can be seen in the three left-hand panels, each for a different METH-treated animals (IDs present at the top left of panels). There was no evidence in this region in any of the three animals for terminal, axonal, or cell body labeling degeneration from FJc labeling in the remaining six panels. Again, as seen in the septum, the most intensely labeled structures were some of the vasculature present. The blue arrows show where the region of high magnification resides in the low magnification panels 
[31] such that faint FJc labeling of other structures such as vasculature can be seen in some cases. Because of this, the fainter images of vasculature, probably endothelium, can be seen in Fig. 4. Note that in this figure, vascular-like structures faintly labeled with FJc appear juxtaposed to activated microglia.

Double labeling to identify both vascular endothelia and microglia was performed to discern whether or not the activated microglia were associated with vasculature in the septum, hippocampus, and thalamus. This was accomplished using antibodies to two markers Cd11b and Iba1 for microglia and RECA-1 antibody for endothelia. Combinations of either TRITC-labeled Iba1 immunoreactivity and DAB-labeled RECA-1 immunoreactivity or DAB-labeled Cd11b immunoreactivity and nitro blue tetrazolium-labeled RECA-1 immunoreactivity to identify vascular endothelium show examples of the many close associations of endothelium with activated microglia in (Fig. 5, septum; Fig. 6, hippocampus; Fig. 7 VL/ VM thalamus) 3 days after METH. In Fig. 6, the purple arrows show a region of very intense activation of microglia surrounding regions where vascular damage is intense in the hippocampus.
Double labeling with DAB-labeled Cd11b immunoreactivity for microglia and blue tetrazolium-labeled RECA1 for vasculature again shows the close association of many/most of the activated microglia with vasculature in both the septum (panels A and B) and the ventrolateral thalamus (panel c, Fig. 8). In panel D, the microglia can be seen double labeled with DAB-labeled Cd11b immunoreactivity and blue tetrazolium-labeled Iba1 immunoreactivity. There is a very close overlap in labeling between the two microglial markers. However, in the activated microglia, the Cd11b tends to be located in the regions of the microglia more distal to the cell body (more filamentous processes) while the IBA1 is more concentrated in the central regions of the microglia.

Figure $9 \mathrm{a}-\mathrm{c}$ shows the morphology of astrocytes surrounding the activated microglia in the dorsal lateral septum, medial anterior hippocampus, and the VL/VM thalamus at 3 days after METH. The swollen and elongated activated DAB-IBA1-labeled microglia are clearly seen under incandescent light (remember from Fig. 8 that the DAB-IBA1 is not labeling the many of distal processes of these activated microglia). The astrocytes in these three brain regions appeared to be somewhat

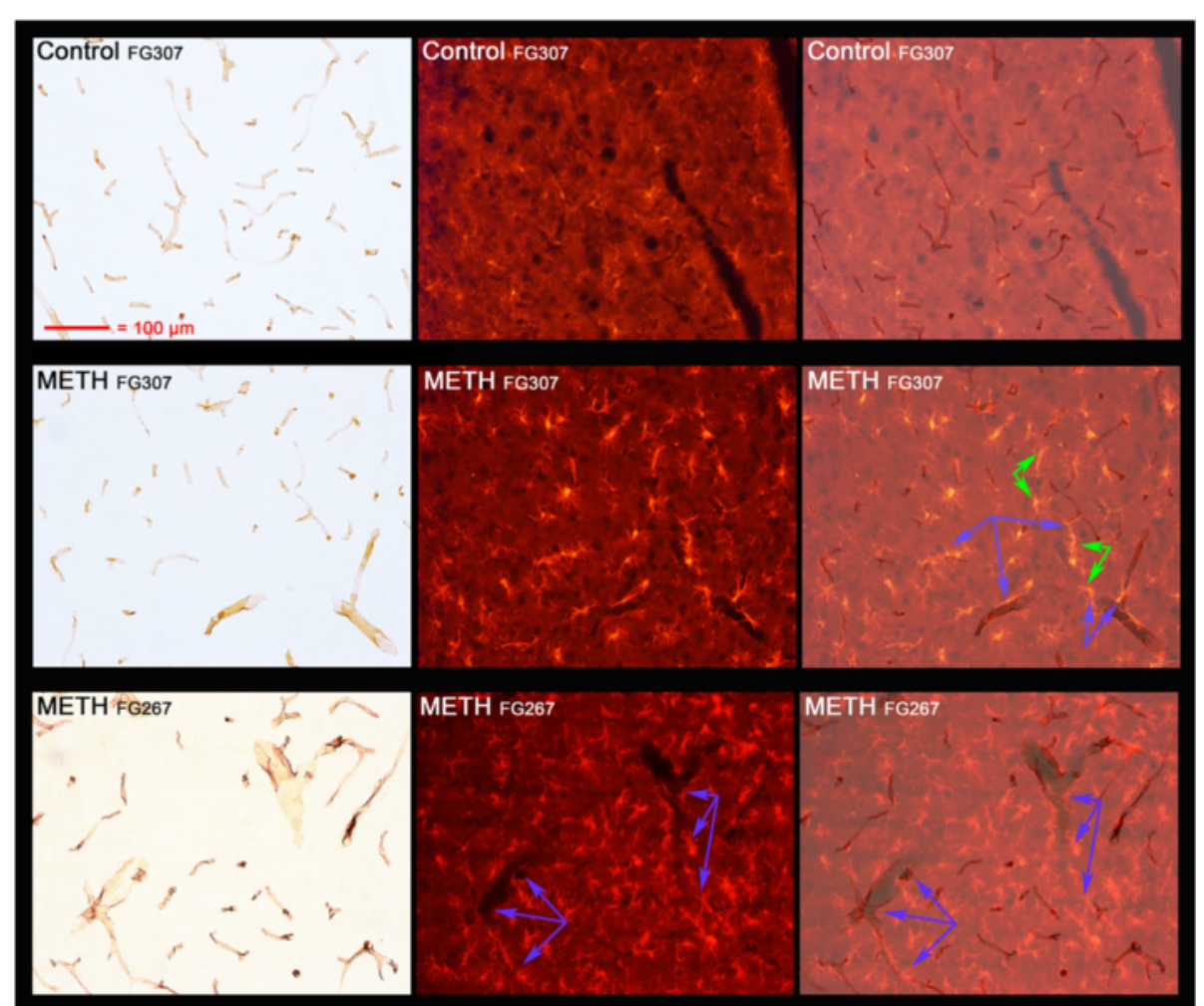

Fig. 5 Activated microglia in septum associated with vasculature after METH. Septal sections from a control and two METH-treated animals were double labeled using DAB labeling of an antibody to RECA1 and TRITC labeling of an antibody to IBA1. The far left panels show the DAB-labeled RECA1 immunoreactivity to vasculature through visible light and the middle panels show the TRITC-labeled IBA1 immunoreactivity to microglia through fluorescent illumination. The three far right panels are a merger of the first two panels. The purple arrows show regions of particular interest (see the "Results" section for details). All panels are of the same magnification 


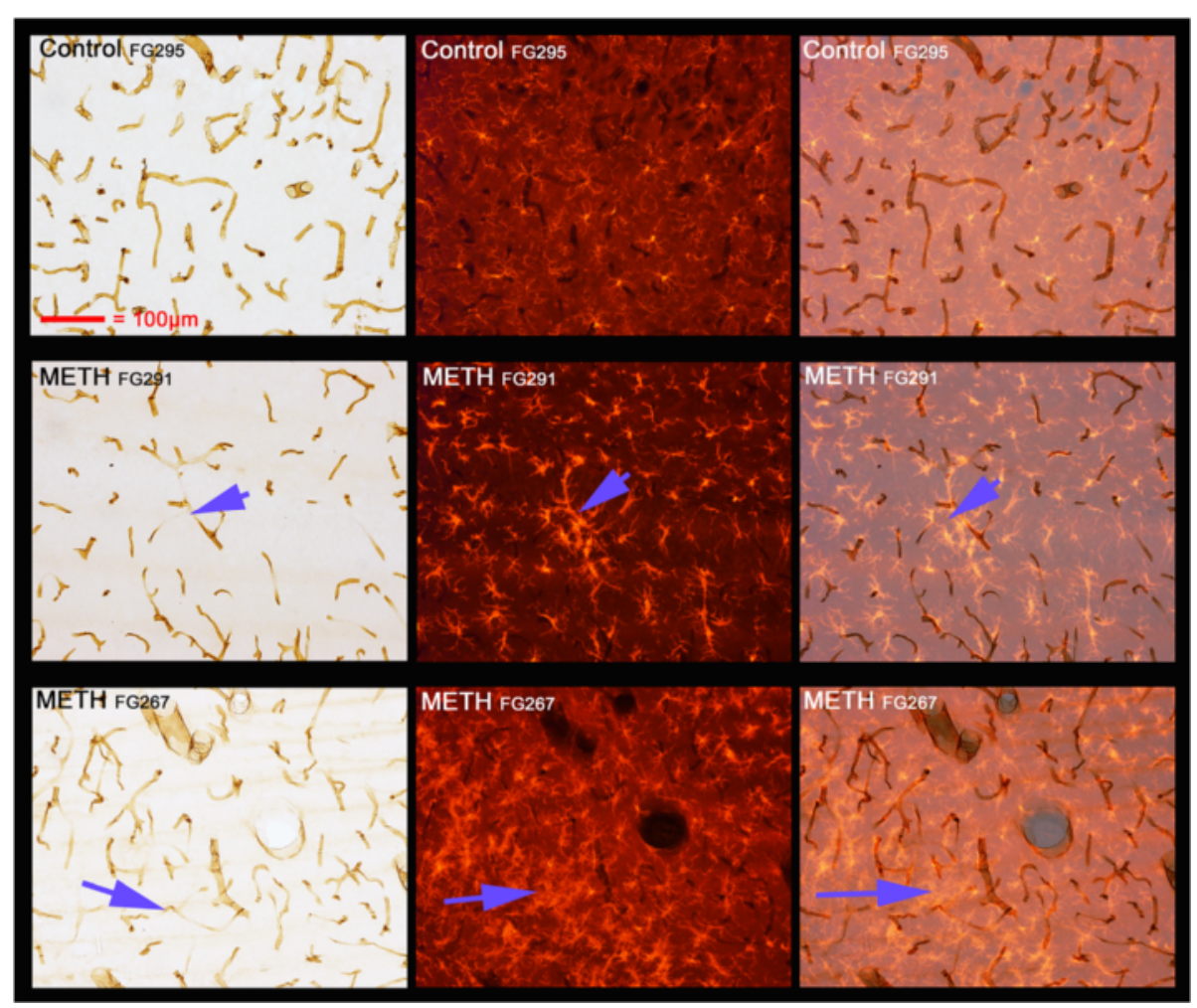

Fig. 6 Activated microglia in hippocampus associated with vasculature after METH. Hippocampal sections from a control and two METH-treated animals were double labeled using DAB labeling of an antibody to RECA1 and TRITC labeling of an antibody to IBA1. The far left panels show the DAB-labeled RECA1 immunoreactivity to vasculature through visible light and the middle panels show the TRITC-labeled IBA1 immunoreactivity to microglia through fluorescent illumination. The far right panels are a merger of the first two panels. The indigo arrows show regions of particular interest (see the "Results" section for details). All panels are of the same magnification

"swollen" and of a slightly different morphology. However, there are no discrete areas within any of the three regions where the morphology of the astrocytes juxtapose to the activated microglia is altered. More extensive data from DAB-GFAP labeling of astrocytes in the septum, hippocampus, and thalamus also indicate that there is a general mild hypertrophy in these regions, but there are no discrete areas within the regions where there is a distinctive change in astrocyte morphology (Additional file 1: Figure S9).

\section{Discussion}

Much has been learned within the last 10 years about microglia including their origin and early integration into the newly developing brain [18, 40], role in both neurological disease $[3,19]$, and response to neurotoxic insults [2]. However, most of the research relating to microglia focuses on their interaction with neurons including the formation of synapses [19], their role in the repair and support of damaged neurons [3, 17], and their proposed responses resulting in adverse neuroinflammation [41]. However, it is clear from very recent research that much is still unknown about the role and importance of microglia in the adult brain under normal conditions or after neurotoxic insult [14, 22, 42-44]. The results of this investigation indicate that activated microglia associate closely with vasculature in regions such as the septum and hippocampus where there is minimal or no evidence of neurodegeneration after METH with the dosing paradigm we employed in the present experiments.

Microglia serve as microsensors of underlying pathology and are activated at sites of neural damage [45]. Previous research looking at microglial responses to METH and amphetamine toxicity have focused on regions of neurodegeneration, both axonal/terminal degeneration or neuronal (cell body) death [9-11, 13, 25, $39,46]$. In such research, we have noted that microglia have appeared to surround vasculature in regions of neurodegeneration in the hippocampus after METH [25]. However, in those studies, the METH exposure was severe enough to produce status epilepticus and significant neurodegeneration of pyramidal neurons (particularly in the CA2 and CA3 regions) as well as evidence of protracted $\mathrm{BBB}$ breakdown in the hippocampus. This left open the possibility that either microglia were merely 


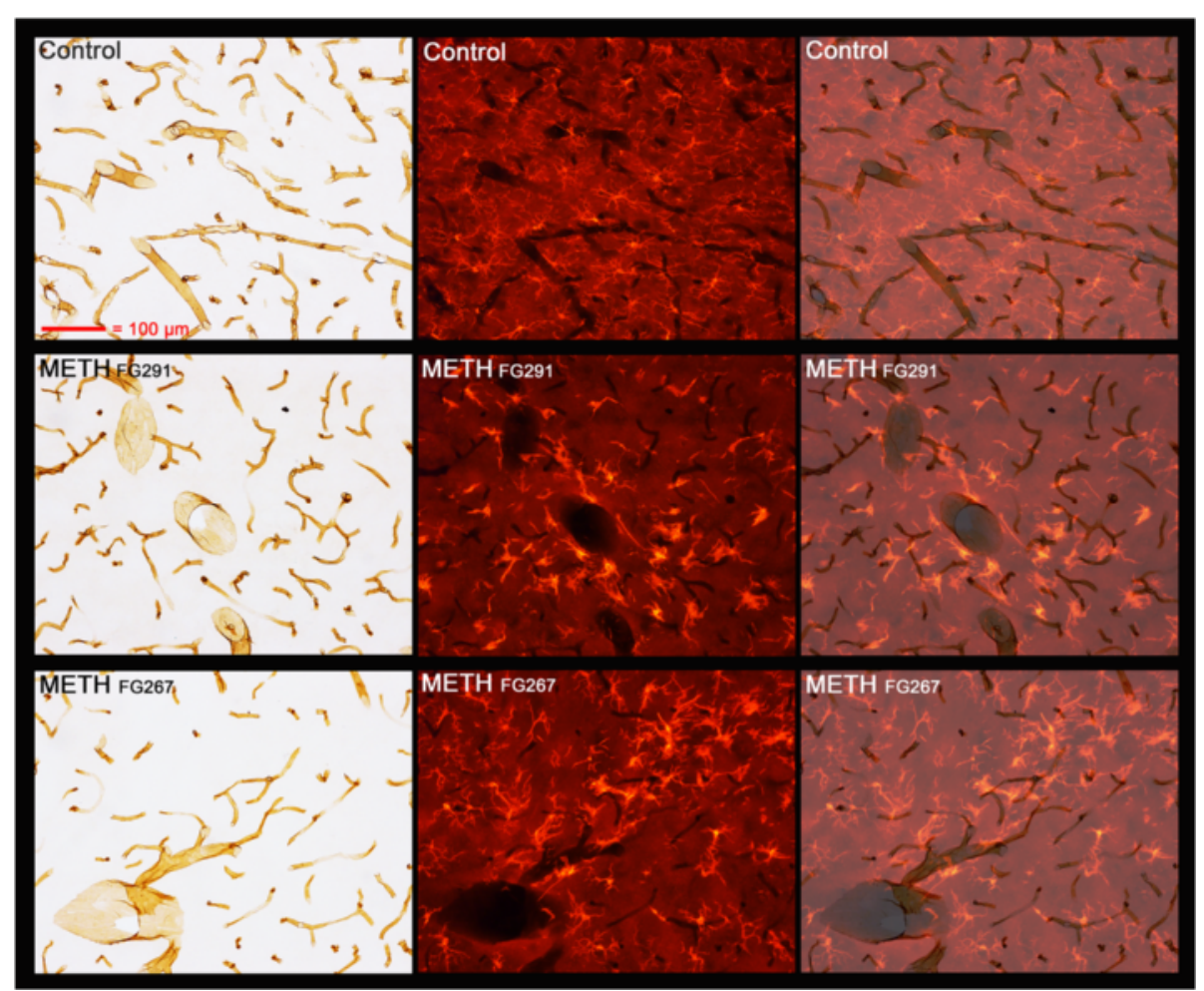

Fig. 7 Activated microglia in the thalamus associated with vasculature after METH. Thalamic sections from a control and two METH-treated animals were double labeled using DAB labeling of an antibody to RECA1 and TRITC labeling of an antibody to IBA1. The far left panels show the DAB-labeled RECA1 immunoreactivity to vasculature through visible light and the middle panels show the TRITC-labeled IBA1 immunoreactivity to microglia through fluorescent illumination. The far right panels are a merger of the first two panels. The indigo arrows show regions of particular interest (see the "Results" section for details). All panels are of the same magnification

responding to intense neurodegeneration or that macrophages had entered the brain in the regions of such severe damage and altered their morphology to resemble microglia. We have also seen similar effects in the thalamus with neurotoxic exposures to amphetamine where microglia appear to surround vasculature in the thalamus (unpublished data, [26]). Although there was little evidence of BBB disruption at this time point after amphetamine in this region, the microglia were observed in regions of significant neurodegeneration.

There is a significant literature related to amphetamine and METH with respect to adverse effects on brain vasculature and how this may be related to hyperthermia and neurotoxicity. Initially, it was observed that even a single dose of METH can produce small discrete regions of vascular leakage in several brain regions of laboratory animals when body temperatures exceed $\approx 41.6{ }^{\circ} \mathrm{C}[28$, 47]. Some of these regions coincided with where amphetamines have been shown to produce neurotoxicity. A later study, using the same dosing paradigm as used in our present study (but substituting amphetamine for METH), observed the same discrete vascular leakage ( $\leq 1.0 \mathrm{~mm}$ in diameter) to occur throughout the entire drug exposure (multiple injections over time) [27]. This leakage lasted only briefly (less than $2 \mathrm{~h}$ ) during the amphetamine administration. However, a single administration/injection of a very high dose of METH or amphetamine $(40 \mathrm{mg} / \mathrm{kg})$ can produce even longer lasting (6 to $12 \mathrm{~h}$ ) and more extensive BBB leakage (in the septum, anterior medial hippocampus, and amygdala nuclei) that results in significant neurodegeneration in these regions $[25,26]$.

Much less is known with respect to how the vascular damage that is produced by amphetamines relates to the hypertension and reduced blood flow that may occur during exposure. A single dose of METH has been shown to produce prolonged reduced perfusion [48], which could certainly adversely affect neuronal function and lead to neurotoxicity. As well, neurotoxic exposure to amphetamine produces changes lasting for at least 1 day in the cortex, meninges, and associated vasculature indicating adverse vascular events have occurred in the cortical surface vasculature $[49,50]$. Others have reported neurotoxicity and microglia activation in the caudate putamen after amphetamines that may be related to dopamine receptor interactions [51,52], effects subsequent to vascular alterations, or damage [53]. Thus, in light of the preceding discussion, it is not entirely 


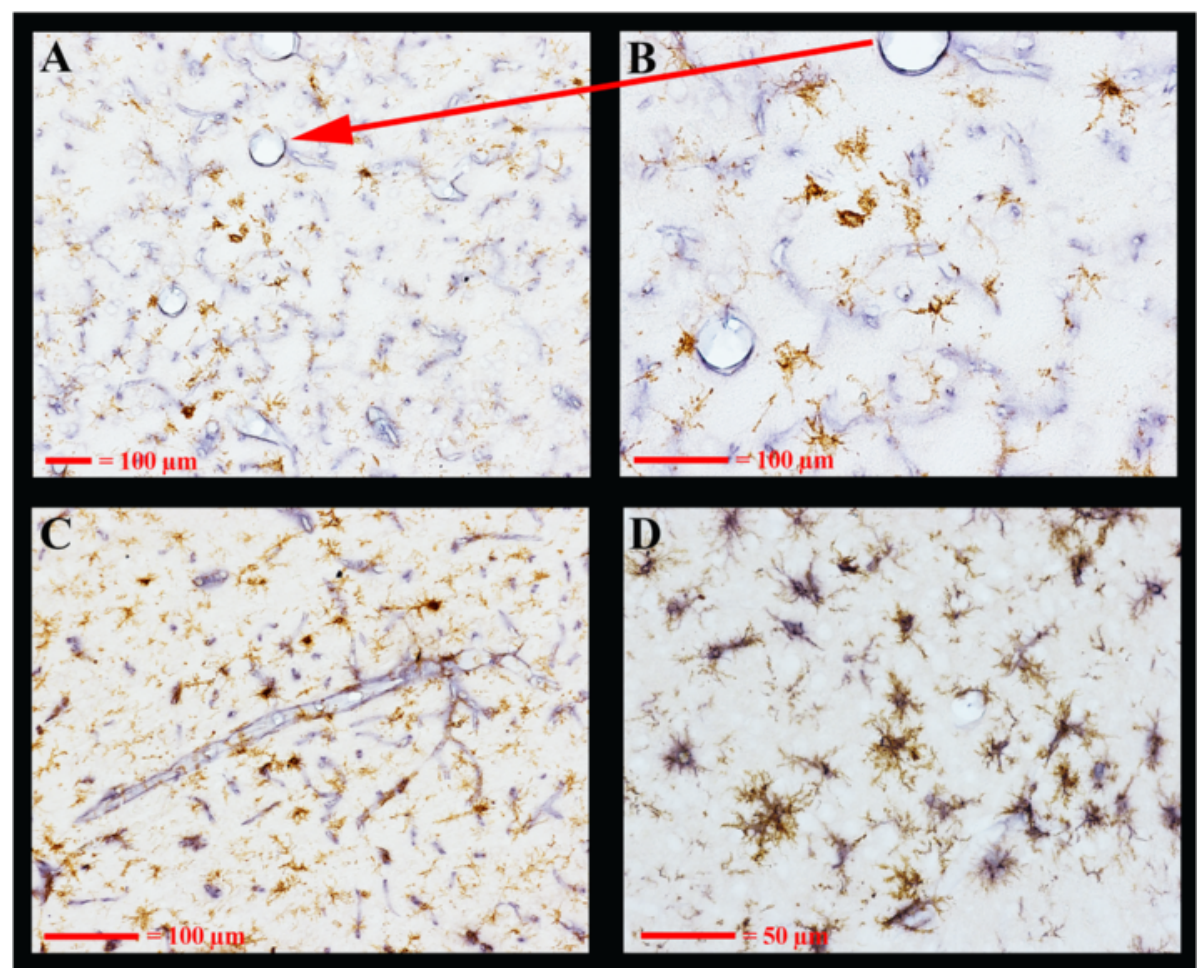

Fig. 8 Activated microglia immunoreactive for Cd11b are shown in association with vasculature after METH. DAB immunolabeling of Cd11 to detect microglia and alkaline phosphatase/tetrazolium-immunolabeling of RECA1 were performed in sections of a METH animal. Shown are the associations of activated microglia with vasculature in the septum in panels $\mathbf{a}$ and $\mathbf{b}$ or the thalamus in panel $\mathbf{c}$ in visible light. Double labeling of microglia with DAB immunolabeling for Cd11b and alkaline phosphatase/tetrazolium-immunolabeling for IBA1 is shown in panel $\mathbf{d}$. Magnification bars in lower left-hand corner

unexpected that microglia might respond to just vascular damage as well as neuronal damage.

Other studies have noted that microglia can be activated in the absence of underlying neurodegeneration, but these effects are still classified as neuroimmune responses (e.g., [14, 24]). Similar to those studies, evidence of neurodegeneration in the septum and hippocampus were not observed in the present study yet activated microglia surrounding vasculature in regions of the septum and hippocampus were seen after METH. Such activation was not seen in other regions of the forebrain except in two animals that had such activated microglia in the piriform cortex and amygdala (data not shown). The morphology of the activated microglia in septum and hippocampus was not that of enlarged spheres with radial processes that can be seen surrounding degenerating neurons but of a swollen, elongated tubular form with seemingly shrunken processes as stained with IBA1. However, double labeling immune-labeling with $\mathrm{Cd} 11 \mathrm{~b}$ indicated that the processes of these activated microglia may not have shrunk in size but that IBA1 levels in the processes were greatly reduced or not present and that $\mathrm{Cd} 11 \mathrm{~b}$ was present distally. As well, there is no evidence of $\mathrm{BBB}$ breakdown as determined by IgG immunereactivity or macrophage invasion in the brain either 1 day or 3 days post METH. Even in the ventrolateral thalamic nuclei and ventromedial thalamic nucleus, where significant neurodegeneration occurred, there were at least an equal number of the activated tubular-shaped microglia closely associated with vasculature.

Pericytes also play an important role in capillary flow, angiogenesis, blood-brain barrier, and immune responses, and they are very similar to microglia in transcriptome expression profile [51,52]. Although some of the IBA1 expressing cells surrounding the vasculature seen in our studies may have been or be pericytes, the vast number observed in the hippocampus, septum, and thalamus would seem to be far too great to be just pericytes. Moreover, there morphology of most of these IBA1-positive cells does not seem to fit that reported for pericytes [53]. However, since there are no clear/certain histological markers that distinguish pericytes from microglia, we cannot definitively determine the percentage of pericytes that might also be IBA1 positive and associated with vasculature in these regions.

It is not clear how the astrocytes are interacting with the microglia in the septum, hippocampus, or thalamus 


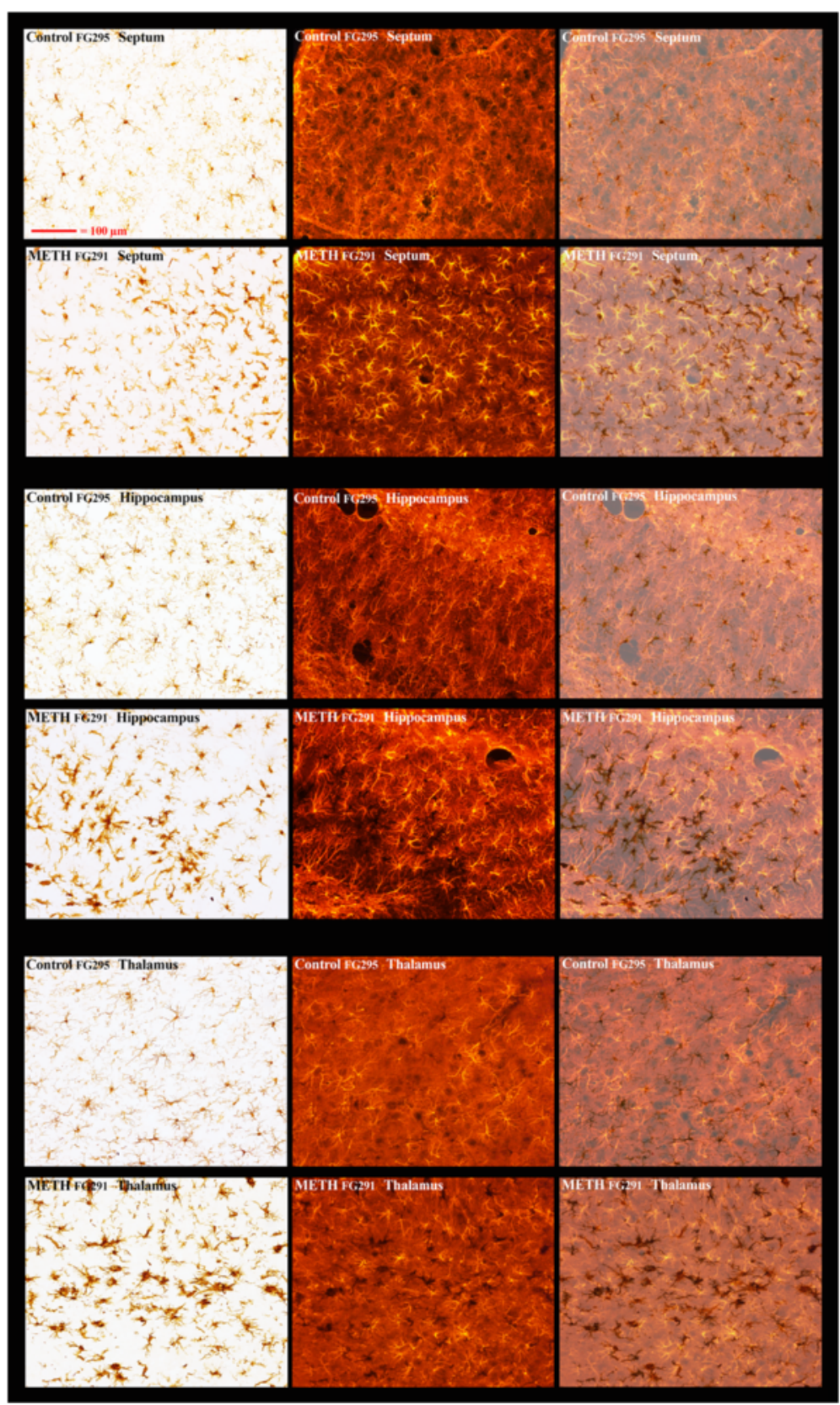

Fig. 9 Astrocyte morphology in regions of METH-induced microglial activation. Dual labeling with DAB in conjunction with IBA1 immunoreactivity and TRITC for GFAP immunolabeling shows the morphology of astrocytes in the septum (top 6 panels), hippocampus (middle 6 panels), and thalamus (bottom 6 panels) in regions in conjunction with activated microglia after METH. The left-hand column of panels shows the IBA1-labeled microglia under incandescent illumination while the center column of panels shows the fluorescent images of the TRITC-labeled astrocytes. The right-hand column of panels is a merger of the incandescent and fluorescent images. All panels are of the same magnification (see the upper left-hand panel for magnification reference)

that are activated 3 days after METH exposure. There appears to be a perceptible modest hypertrophy of the astrocytes in these three regions, but it is region-wide and not in conjunction with the specific areas of microglial activation surrounding the vasculature. Areas with modest toxicant-induced neural damage can result in increases in levels of the astrocytic protein, GFAP, in the absence of an apparent astrocytic hypertrophy [38], an effect often preceded by microglia activation in the same region [38]. Thus, it is possible that a small degree of METH-induced damage, undetectable via FJc, in these areas, is responsible for the observed modest astrocyte 
responses. However, they do not explain the close responses/associations of microglia with specific vasculature and not neurons.

\section{Conclusions}

The simplest and, in our opinion, the most logical explanation for the presence of activated microglia surrounding the vasculature in two discrete regions of the brain where there is no neurodegeneration would be that the microglia are responding to METH-induced vascular damage. This was facilitated by multiple episodes of hyperthermia (an exposure comparable to heat stroke) and hypertension that was produced by the METH exposure. It thus follows that when more episodes of hyperthermia occur, the vascular damage and microglial activation in the septum and hippocampus are more likely to occur and more intense. The METH exposure we employed only produces transient vascular disruptions lasting less than 1 to $2 \mathrm{~h}$ and is most commonly seen in the thalamus, septum, piriform cortex, and medial ventral hippocampus [26-28]. This may be sufficient enough to allow cytokines normally excluded from the brain or the damage-associated proteins (DAMPs) produced by METH exposure [54] to enter the brain and activate the microglia. On the other hand, it may be that adversely affected brain vascular endothelia are directly signaling the microglia through some unknown messenger(s) signaling pathway. If this is the case, then the term cerebrovascular-induced brain inflammation would be a more appropriate characterization rather than describing it as neuroinflammation. It follows that detection of such activated microglia several days after an "adverse" event occurring in brain could be used as a biomarker indicating that some vascular damage or "irritation" had occurred.

\section{Additional files}

Additional file 1: Figure S1A. DAB-lba1 labeled microglia activation in the septum at 3 days after METH exposure. Figure S1B. DAB-lba1 labeled microglia in the septum and hippocampus at 1day after METH exposure. Figure S2A. DAB-lba1 labeled microglia in the hippocampus at 3 days after METH exposure. Figure S2B. DAB-Iba1 labeled microglia in the cudate/putamen at 1 day after METH exposure. Figure S3A. Coincidence of degenerating (FJC+) neurons and activated microglia in the thalamus at 3 days after METH. Figure S3B. FJc-labeling in the septum at 3 days after METH exposure. Figure S4. FJc-labeling in the medial hippocampus at 3 days after METH exposure. Figure S9. DAB-GFAP labeled astrocytes in the medial hippocampus at 3 days after METH exposure. (PDF $15680 \mathrm{~kb}$ )

\section{Abbreviations}

AMPH: amphetamine; a.k.a. Cd11b: integrin, alpha M (complement component 3 receptor 3 subunit), integrin, alpha M (complement component 3 receptor 3 subunit) a.k.a.ltgam; DAB: diaminobenzedine; FITC: fluorescein isothiocyanate; FJC: Fluoro-Jade C; allograft inflammatory factor 1: Aif1 a.k.a Iba1; METH: methamphetamine; RECA-1: rat endothelial cell antigen-1; TRITC: tetramethylrhodamine-isothiocyanate.

\section{Competing interests}

The authors have no financial or personal competing interests to report. However, see the following regarding the FDA disclaimer-The contents of this manuscript do not necessarily reflect the views and policies of the U.S. Food and Drug Administration, nor does the mention of trade names or commercial products constitute endorsement or a recommendation for use.

\section{Authors' contributions}

$J B$ was involved in all aspects of the submission, including animal handling and dosing and generation of immunohistochemical data. SS participated in the aspects of the study design, data interpretation, the generation of immunohistochemical data, and manuscript preparation and submission. KT participated in the aspects of the study design, primarily responsible for animal handling and dosing and the generation of immunohistochemical data. JH participated in obtaining support for the study, study design, and manuscript preparation and submission. DM and JO were involved in the study design, data interpretation, and manuscript preparation. All authors read and approved the final manuscript.

\section{Acknowledgement}

This study was supported by FDA protocol E7519.

\section{Author details}

1Division of Neurotoxicology, National Center for Toxicology/FDA, Jefferson, AR 72079, USA. ${ }^{2}$ Center for Drug Evaluation and Research/FDA, Silver Spring, MD 20993, USA. ${ }^{3}$ Health Effects Laboratory Division, Centers for Disease Control and Prevention, National Institute for Occupational Safety and Health, Morgantown, WV 26505, USA. ${ }^{4}$ National Center for Toxicological Research/FDA, 3900 NCTR Road, HFT-132, Jefferson, AR 72079, USA.

Received: 31 January 2016 Accepted: 3 March 2016

Published online: 12 March 2016

\section{References}

1. Streit WJ. The role of microglia in brain injury. Neurotoxicology. 1996;17:671-8.

2. Streit WJ. Microglia and the response to brain injury. Ernst Schering Res Found Workshop. 2002;39:11-24.

3. Streit WJ. Microglial senescence: does the brain's immune system have an expiration date? Trends Neurosci. 2006;29:506-10.

4. Nadler JV, Perry BW, Gentry C, Cotman CW. Degeneration of hippocampal CA3 pyramidal cells induced by intraventricular kainic acid. J Comp Neurol. 1980;192:333-59.

5. Lipton SA. HIV-related neurotoxicity. Brain Pathol. 1991:1:193-9.

6. Appel NM, Rapoport SI, O'Callaghan JP. Sequelae of parenteral domoic acid administration in rats: comparison of effects on different anatomical markers in brain. Synapse. 1997;25:350-8.

7. Beadell NC, Thompson EM, Delashaw JB, Cetas JS. The deleterious effects of methamphetamine use on initial presentation and clinical outcomes in aneurysmal subarachnoid hemorrhage. J Neurosurg. 2012;117:781-6.

8. Ransohoff RM, Schafer D, Vincent A, Blachere NE, Bar-Or A. Neuroinflammation: ways in which the immune system affects the brain. Neurotherapeutics. 2015:12:896-909.

9. Hess A, Desiderio C, McAuliffe WG. Acute neuropathological changes in the caudate nucleus caused by MPTP and methamphetamine: immunohistochemical studies. J Neurocytol. 1990;19:338-42.

10. LaVoie MJ, Card JP, Hastings TG. Microglial activation precedes dopamine terminal pathology in methamphetamine-induced neurotoxicity. Exp Neurol. 2004;187:47-57.

11. Bowyer JF, Peterson SL, Rountree RL, Tor-Agbidye J, Wang GJ. Neuronal degeneration in rat forebrain resulting from D-amphetamine-induced convulsions is dependent on seizure severity and age. Brain Res. 1998;809:77-90.

12. Eisch AJ, Schmued LC, Marshall JF. Characterizing cortical neuron injury with Fluoro-Jade labeling after a neurotoxic regimen of methamphetamine. Synapse. 1998;30:329-33.

13. Schmued LC, Bowyer JF. Methamphetamine exposure can produce neuronal degeneration in mouse hippocampal remnants. Brain Res. 1997;759:135-40. 
14. O'Callaghan JP, Kelly KA, VanGilder RL, Sofroniew MV, Miller DB. Early activation of STAT3 regulates reactive astrogliosis induced by diverse forms of neurotoxicity. PLoS One. 2014;9:e102003.

15. O'Callaghan JP, Sriram K, Miller DB. Defining "neuroinflammation". Ann N Y Acad Sci. 2008;1139:318-30.

16. Sriram K, Miller DB, O'Callaghan JP. Minocycline attenuates microglial activation but fails to mitigate striatal dopaminergic neurotoxicity: role of tumor necrosis factor-alpha. J Neurochem. 2006;96:706-18.

17. Aguzzi A, Barres BA, Bennett ML. Microglia: scapegoat, saboteur, or something else? Science. 2013;339:156-61.

18. Ransohoff RM, Cardona AE. The myeloid cells of the central nervous system parenchyma. Nature. 2010;468:253-62

19. Prinz M, Priller J. Microglia and brain macrophages in the molecular age: from origin to neuropsychiatric disease. Nat Rev Neurosci. 2014;15:300-12.

20. Prinz M, Tay TL, Wolf $Y$, Jung S. Microglia: unique and common features with other tissue macrophages. Acta Neuropathol. 2014;128:319-31.

21. Corps KN, Roth TL, McGavern DB. Inflammation and neuroprotection in traumatic brain injury. JAMA Neurol. 2015;72:355-62.

22. Elmore MR, Najafi AR, Koike MA, Dagher NN, Spangenberg EE, Rice RA, et al. Colony-stimulating factor 1 receptor signaling is necessary for microglia viability, unmasking a microglia progenitor cell in the adult brain. Neuron. 2014;82:380-97.

23. Hanisch UK, Kettenmann H. Microglia: active sensor and versatile effector cells in the normal and pathologic brain. Nat Neurosci. 2007;10:1387-94.

24. Buttini M, Limonta S, Boddeke HW. Peripheral administration of lipopolysaccharide induces activation of microglial cells in rat brain. Neurochem Int. 1996:29:25-35.

25. Bowyer JF, Ali S. High doses of methamphetamine that cause disruption of the blood-brain barrier in limbic regions produce extensive neuronal degeneration in mouse hippocampus. Synapse. 2006;60:521-32.

26. Bowyer JF, Thomas MT, Schmued LC, Ali SF. Brain region-specific neurodegenerative profiles showing the relative importance of amphetamine dose, hyperthermia, seizures and the blood-brain barrier. Ann NY Acad Sci. 2008;1139:127-39.

27. Bowyer JF, Tranter KM, Sarkar S, Raymick J, Hanig JP, Schmued LC. Systemic administration of fluoro-gold for the histological assessment of vascular structure, integrity and damage. Curr Neurovasc Res. 2014;11:31-47.

28. Kiyatkin EA, Brown PL, Sharma HS. Brain edema and breakdown of the blood-brain barrier during methamphetamine intoxication: critical role of brain hyperthermia. Eur J Neurosci. 2007;26:1242-53.

29. Sonsalla PK, Nicklas WJ, Heikkila RE. Role for excitatory amino acids in methamphetamine-induced nigrostriatal dopaminergic toxicity. Science. 1989;243:398-400

30. Paxinos, G. and Watson, C. The rat brain in stereotaxix coordinates. Volume 2, Academic Press, San Diego, CA.

31. Schmued LC, Stowers CC, Scallet AC, Xu L. Fluoro-Jade C results in ultra high resolution and contrast labeling of degenerating neurons. Brain Res. 2005;1035:24-31.

32. Bowyer JF, Schmued LC. Fluoro-Ruby labeling prior to an amphetamine neurotoxic insult shows a definitive massive loss of dopaminergic terminals and axons in the caudate-putamen. Brain Res. 2006;1075:236-9.

33. Cass WA, Manning MW, Dugan MT. Effects of neurotoxic doses of methamphetamine on potassium and amphetamine evoked overflow of dopamine in the striatum of awake rats. Neurosci Lett. 1998;248:175-8.

34. Ricaurte GA, McCann UD. Neurotoxic amphetamine analogues: effects in monkeys and implications for humans. Ann N Y Acad Sci. 1992;648:371-82.

35. Ricaurte GA, Schuster CR, Seiden LS. Long-term effects of repeated methylamphetamine administration on dopamine and serotonin neurons in the rat brain: a regional study. Brain Res. 1980;193:153-63.

36. Ahmed Z, Shaw G, Sharma VP, Yang C, McGowan E, Dickson DW. Actinbinding proteins coronin-1a and IBA-1 are effective microglial markers for immunohistochemistry. J Histochem Cytochem. 2007:55:687-700.

37. McCann MJ, O'Callaghan JP, Martin PM, Bertram T, Streit WJ. Differential activation of microglia and astrocytes following trimethyl tin-induced neurodegeneration. Neuroscience. 1996;72:273-81.

38. Brock TO, O'Callaghan JP. Quantitative changes in the synaptic vesicle proteins synapsin I and p38 and the astrocyte-specific protein glial fibrillary acidic protein are associated with chemical-induced injury to the rat central nervous system. J Neurosci. 1987;7:931-42.

39. Thomas DM, Walker PD, Benjamins JA, Geddes TJ, Kuhn DM Methamphetamine neurotoxicity in dopamine nerve endings of the striatum is associated with microglial activation. J Pharmacol Exp Ther. 2004;311:1-7.

40. Katsumoto A, Lu H, Miranda AS, Ransohoff RM. Ontogeny and functions of central nervous system macrophages. J Immunol. 2014;193:2615-21.

41. Heppner FL, Ransohoff RM, Becher B. Immune attack: the role of inflammation in Alzheimer disease. Nat Rev Neurosci. 2015;16:358-72.

42. Rice RA, Spangenberg EE, Yamate-Morgan H, Lee RJ, Arora RP, Hernandez $M X$, et al. Elimination of microglia improves functional outcomes following extensive neuronal loss in the hippocampus. J Neurosci. 2015;35:9977-89.

43. Kelly KA, Miller DB, Bowyer JF, O'Callaghan JP. Chronic exposure to corticosterone enhances the neuroinflammatory and neurotoxic responses to methamphetamine. J Neurochem. 2012;122:995-1009

44. O'Callaghan JP, Kelly KA, Locker AR, Miller DB, Lasley SM. Corticosterone primes the neuroinflammatory response to DFP in mice: potential animal model of Gulf War IIIness. J Neurochem. 2015;133:708-21.

45. Kreutzberg GW. Microglia: a sensor for pathological events in the CNS. Trends Neurosci. 1996:19:312-8.

46. Thomas DM, Kuhn DM. MK-801 and dextromethorphan block microglial activation and protect against methamphetamine-induced neurotoxicity. Brain Res. 2005;1050:190-8.

47. Kiyatkin EA. Brain hyperthermia during physiological and pathological conditions: causes, mechanisms, and functional implications. Curr Neurovasc Res. 2004;1:77-90.

48. Polesskaya O, Silva J, Sanfilippo C, Desrosiers T, Sun A, Shen J, et al. Methamphetamine causes sustained depression in cerebral blood flow. Brain Res. 2011:1373:91-100.

49. Bowyer JF, Patterson TA, Saini UT, Hanig JP, Thomas M, Camacho L, et al. Comparison of the global gene expression of choroid plexus and meninges and associated vasculature under control conditions and after pronounced hyperthermia or amphetamine toxicity. BMC Genomics. 2013;14:147.

50. Thomas M, George NI, Patterson TA, Bowyer JF. Amphetamine and environmentally induced hyperthermia differentially alter the expression of genes regulating vascular tone and angiogenesis in the meninges and associated vasculature. Synapse. 2009;63:881-94

51. Kamouchi M, Ago T, Kitazono T. Brain pericytes: emerging concepts and functional roles in brain homeostasis. Cell Mol Neurobiol. 2011;31:175-93.

52. Bell RD, Winkler EA, Sagare AP, Singh I, LaRue B, Deane R, et al. Pericytes control key neurovascular functions and neuronal phenotype in the adult brain and during brain aging. Neuron. 2010;68:409-27.

53. Sarkar S, Schmued L. In vivo administration of fluorescent dextrans for the specific and sensitive localization of brain vascular pericytes and their characterization in normal and neurotoxin exposed brains. Neurotoxicology. 2012;33:436-43

54. Levi MS, Patton RE, Hanig JP, Tranter KM, George NI, James LP, et al. Serum myoglobin, but not lipopolysaccharides, is predictive of $\mathrm{AMPH}$-induced striatal neurotoxicity. Neurotoxicology. 2013;37C:40-50.

\section{Submit your next manuscript to BioMed Central and we will help you at every step:}

- We accept pre-submission inquiries

- Our selector tool helps you to find the most relevant journal

- We provide round the clock customer support

- Convenient online submission

- Thorough peer review

- Inclusion in PubMed and all major indexing services

- Maximum visibility for your research

Submit your manuscript at www.biomedcentral.com/submit
) Biomed Central 